\title{
The flow field structure of highly stabilized partially premixed flames in a concentric flow conical nozzle burner with coflow
}

\author{
A.M. Elbaz ${ }^{\mathrm{a}, \mathrm{b}, *}$, M.F. Zayed ${ }^{\mathrm{c}}$, M. Samy ${ }^{\mathrm{d}}$, W.L. Roberts ${ }^{\mathrm{a}}$, M.S. Mansour ${ }^{\mathrm{c}, \mathrm{e}}$ \\ ${ }^{a}$ Clean Combustion Research Center, King Abdullah University for Science and Technology, Saudi Arabia \\ ${ }^{\mathrm{b}}$ Mechanical Power Engineering Department, Helwan University, Cairo, Egypt \\ ${ }^{\mathrm{c}}$ Mechanical Power Engineering Department, Cairo University, Giza, Egypt \\ ${ }^{\mathrm{d}}$ National Institute of Laser Enhanced Sciences, Cairo University, Giza, Egypt \\ e Mechanical Engineering Department, The American University in Cairo, Egypt
}

\section{A R T I C L E I N F O}

\section{Article history:}

Received 23 August 2015

Accepted 24 August 2015

Available online $\mathrm{xxxx}$

\section{Keywords:}

Partially premixed

Flames

Combustion

Stability

Flow field

\begin{abstract}
A B S T R A C T
The stability limits, the stabilization mechanism, and the flow field structure of highly stabilized partially premixed methane flames in a concentric flow conical nozzle burner with air co-flow have been investigated and presented in this work. The stability map of partial premixed flames illustrates that the flames are stable between two extinction limits. A low extinction limit when partial premixed flames approach non-premixed flame conditions, and a high extinction limit, with the partial premixed flames approach fully premixed flame conditions. These two limits showed that the most stable flame conditions are achieved at a certain degree of partial premixed. The stability is improved by adding air co-flow. As the air co-flow velocity increases the most stable flames are those that approach fully premixed. The turbulent flow field of three flames at $0,5,10 \mathrm{~m} / \mathrm{s}$ co-flow velocity are investigated using Stereo Particle Image Velocimetry (SPIV) in order to explore the improvement of the flame stability due to the use of air co-flow. The three flames are all at a jet equivalence ratio $\left(\Phi_{j}\right)$ of 2 , fixed level of partial premixing and jet Reynolds number $\left(R e_{j}\right)$ of 10,000 . The use of co-flow results in the formation of two vortices at the cone exit. These vortices act like stabilization anchors for the flames to the nozzle tip. With these vortices in the flow field, the reaction zone shifts toward the reduced turbulence intensity at the nozzle rim of the cone. Interesting information about the structure of the flow field with and without co-flow are identified and reported in this work.
\end{abstract}

(c) 2015 Elsevier Inc. All rights reserved.

\section{Introduction}

Partially premixed combustion is prevalent in a wide range of applications for combustion devices: e.g., diesel engines, direct injection stratified charge engines, gas turbines, and some industrial burners. A partially premixed flame (PPF) is described as a hybrid flame possessing characteristics of both premixed and non-premixed flames covering a wide range of flames including "double," "triple" and "edge" flames where mixture is compositionally inhomogeneous. PPF's contain multiple reaction zones which are spatially separated but actually coupled through turbulence-chemistry interactions between them, and the flame structure is strongly dependent upon these interactions [1]. Partially premixed flames are likely to be more stable than

\footnotetext{
* Corresponding author at: Clean Combustion Research Center, King Abdullah University for Science and Technology, Saudi Arabia

E-mail address: ayman.elhagrasy@kaust.edu.sa (A.M. Elbaz).
}

premixed or non-premixed flames [2-4]. Different techniques have been used to stabilize these flames at the nozzle exit. Examples of these techniques include the partially premixed flames [2-5], the swirl flame [6-9], the pilot flame for non-premixed [10,11]; premixed [12], and flame holder [13,14].

A Concentric Flow Conical Nozzle ( $\mathrm{CFCN}$ ) burner has been developed and modified by Mansour $[4,15]$ in order to create well-controlled and highly stabilized partially premixed flames. The higher stability characteristic of the CFCN burner, as compared to a nozzle jet burner, is due to the conical shape of the nozzle and control of the level of partial premixing. The effects of the degree of partial premixing, the cone angle and the jet equivalence ratio on the flame stability and structure have previously been investigated [16]. Measurements and numerical study were conducted inside and downstream the conical nozzle in order to explore the stabilization mechanism and investigate the flame structure of partially premixed flames [17,18] under different equivalence ratios and degrees of partial premixed. They $[17,18]$ concluded that the stabilization mechanism of the flames within the CFCN for high 


\section{Nomenclature}

Re Reynolds number

$R e_{j} \quad$ Reynolds number for the jet mixture

$V \quad$ axial velocity at any radial location

$V_{a} \quad$ axial air velocity at the exit of air tube

$V_{c} \quad$ co-flow exit velocity

$V_{f} \quad$ fuel jet velocity at exit of the fuel tube

$V_{j} \quad$ mixture velocity at the exit of the outer tube
$V_{c e n} \quad$ centerline velocity

$V_{o} \quad$ velocity at the exit of the nozzle

$V_{r m s} \quad$ rms of axial velocity

$V_{\text {cen rms }}$ rms of the axial velocity at the centerline

$r \quad$ radial distance from centerline

$Z \quad$ vertical axial distance

$\Phi_{j} \quad$ mixture equivalence ratio
Reynolds number flames was due to a stream of air being entrained along the conical nozzle wall and heated by the flame before being entrained into the early region of the flame. Additionally, they observed a recirculation zone of hot combustion products at the conical nozzle base. Thus the conical nozzle provides a good stabilization environment. In addition, an optimum level of partial premixing for the highest stability can be achieved in this burner [16]. The temperature and gas species concentrations inside and downstream the nozzle are investigated by Elbaz [19] for Liquid Petroleum Gas (LPG) partial premixed flames. This work [19] showed the same conclusions regarding the flame stability mechanism.

Large capacity burners are usually equipped with a blower for air supply. The effect of co-flow on the partial premixed flames stabilized in the conical nozzle has not yet been investigated. In addition, modeling of the burner requires quantitative data with well-known boundary conditions. With this incentive the main objective of this study is to investigate the effects of the co-flow on the flame stability and the flow field structure of turbulent partially premixed flames within the CFCN burner. The stability mapped is scanned for different degree of partial premixing and different co-flow velocities. The turbulent flow fields for partially premixed flames with and without co-flow are recorded and investigated in this work using a stereo PIV set up.

\section{Experimental setup and measurement technique}

\subsection{Burner}

The process of introducing partial premixing (inhomogeneity) is rather simple and involves two concentric pipes where the inner pipe is recessed to various distances upstream of the jet exit plane. Air passes through the inner tube while fuel (methane) passes through the annular gap between the inner and the outer tubes, as shown in Fig. 1a. The inner diameter of the inner tube " $d$ " is $4 \mathrm{~mm}$ with $1 \mathrm{~mm}$ lip thickness, while the inner diameter of the outer tube " $D$ " is $9.7 \mathrm{~mm}$ with $1.5 \mathrm{~mm}$ lip thickness.

The mixing between the air and the fuel starts at the exit of the inner tube and continuous downstream through the premixing length " $L$," as shown in Fig. 1b. The level of partial premixing varies by varying the distance $L$. The parameter $L / D$ is used to describe several states of partial premixing, when the inner tube is sufficiently recessed $(L / D>30)$, the fuel and air become homogeneously mixed and the mixture is considered fully premixed. The other extreme, when both tubes are flush $(L / D=0)$, represents the nonpremixed limit. The interesting partial premixed flame conditions lie between these two limits where the issuing mixtures are rather compositionally inhomogeneous. To stabilize the issuing flames, a divergent conical nozzle with a half cone angle of $26^{\circ}$ is used at the exit of the outer tube. The length of the cone is $65 \mathrm{~mm}$ with a $73 \mathrm{~mm}$ exit diameter. The burner is located in a cylindrical wind tunnel, as shown in Fig. 1b, that provides a uniform co-flowing air from a blower. The co-flowing air velocity in the present measurements varies from $0 \mathrm{~m} / \mathrm{s}$ to $15 \mathrm{~m} / \mathrm{s}$ with steps of $5 \mathrm{~m} / \mathrm{s}$. The case of $0 \mathrm{~m} / \mathrm{s}$ co-flow velocity is the reference case of the absence of co-flow.

\subsection{Experimental technique}

A stereo PIV system is used to measure the flow field structure of partial premixed flames issuing from CFCN burner. The stereoPIV system is based on a dual cavity, diode-pumped, solid state Neodymium-doped Yttrium Lithium Fluorides (Nd:YLF) laser (LDY 300 Series) and a pair of high-speed complementary metaloxide-semiconductor (CMOS) cameras (LaVision, Image Pro HS $4 \mathrm{M})$. The laser beam wavelength is $527 \mathrm{~nm}$ and its power is $35 \mathrm{~W}$ per head at a repetition rate up to $10 \mathrm{kHz}$, with $9 \mathrm{~ns}$ pulse duration. The laser beam was formed into a sheet with a $0.5-\mathrm{mm}$ waist along the jet centerline using three cylindrical lenses. The air and fuel jets were both seeded with titanium dioxide $\left(\mathrm{TiO}_{2}\right)$ particles with a nominal diameter of $0.5 \mu \mathrm{m}$ via two seeding fluidized bed units. Mie scattered light from the particle-laden flow was collected using a $105 \mathrm{~mm}, \mathrm{f} / 4$ objective (Nikon UV Micro-Nikkor) equipped with a $527 \mathrm{~nm}$ band pass filter. Two cameras were mounted equidistant from the centerline of the burner with an angle of separation of $35^{\circ}$. The camera was operated in twoframe burst mode at $500 \mathrm{fps}$. Image-blur due to off-axis defocusing was corrected using Scheimpflug adaptors between the objectives and the cameras. Perspective distortion was corrected using a dual plane, three-dimensional imaging target (LaVision type 22). Image de-warping between the two camera images was corrected using the image correction and distortion function. Also, with the selfcalibration function, the coordinate system and the camera calibration of this stereoscopic PIV setup were adjusted so that the $Y=0 \mathrm{~mm}$ plane (see Fig. 1 for coordinates) was adjusted exactly in the middle of the laser light sheet. Vector fields were computed from particle image spatial cross correlations using the La Vision Davis 8.1 software package. An adaptive multi-pass vector evaluation technique was used, with interrogation boxes ranging from 128 pixels to 16 pixels, with $50 \%$ overlap resulting in spatial resolution and vector spacing of approximately $0.5 \times 0.5 \mathrm{~mm}$ and $0.25 \mathrm{~mm}$, respectively. The final velocity vector fields were smoothed with a $3 \times 3$ vector moving average filter for subsequent analysis.

\section{Results and discussion}

\subsection{Stability limits, selected flames, and flame appearance}

The stability characteristics of an earlier version of the CFCN burner indicated that partially premixed flames are more stable than premixed or non-premixed flames, and that the level of partial premixing is a critical flame stability parameter [4]. The flames created within the CFCN burner exhibit high stability levels because of the conical shape of the nozzle and the degree of partial premixing. The stability limits that were reported in 


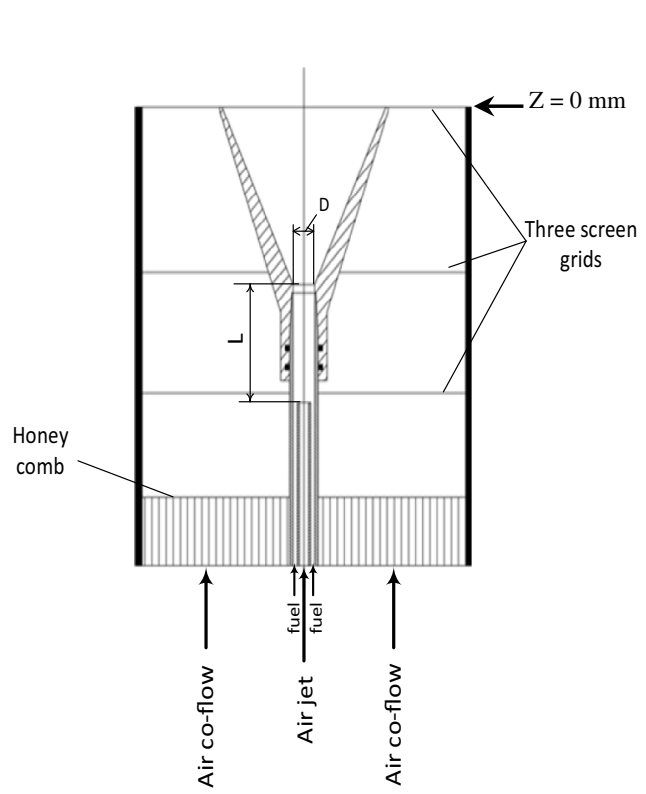

(a)

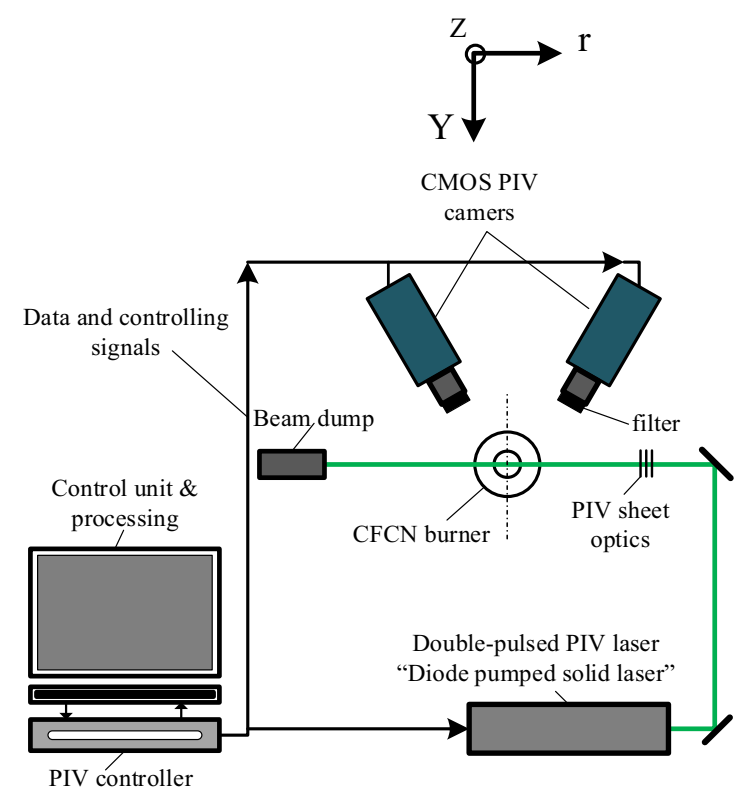

(b)

Fig. 1. (a) A systematic of the CFCN burner with co-flow arrangements, (b) stereo PIV setup.

Refs. [4,15-19] for different flame conditions were without co-flow stabilization. In the present work, we introduced a co-flow parameter in order to extend this study and observe its effect on the flame stabilization mechanism within the CFCN burner at different degree of partial premixing.

Under a fixed co-flow velocity, the stability limit was obtained for a certain overall jet equivalence ratio, where the premixing length $L$ is changed towards the premixed and non-premixed flames conditions until the flames totally blow off. The $L / D$ limit at extinction is recorded for each jet Reynolds number $R e_{j}$ $\left(R e_{j}\right.$ : calculated based on the exit mixture condition of the outer burner tube). Consequently, the relation between jet Reynolds number and the two extinction limits are recorded and plotted for a specific jet equivalence ratio. The experiments, for flames

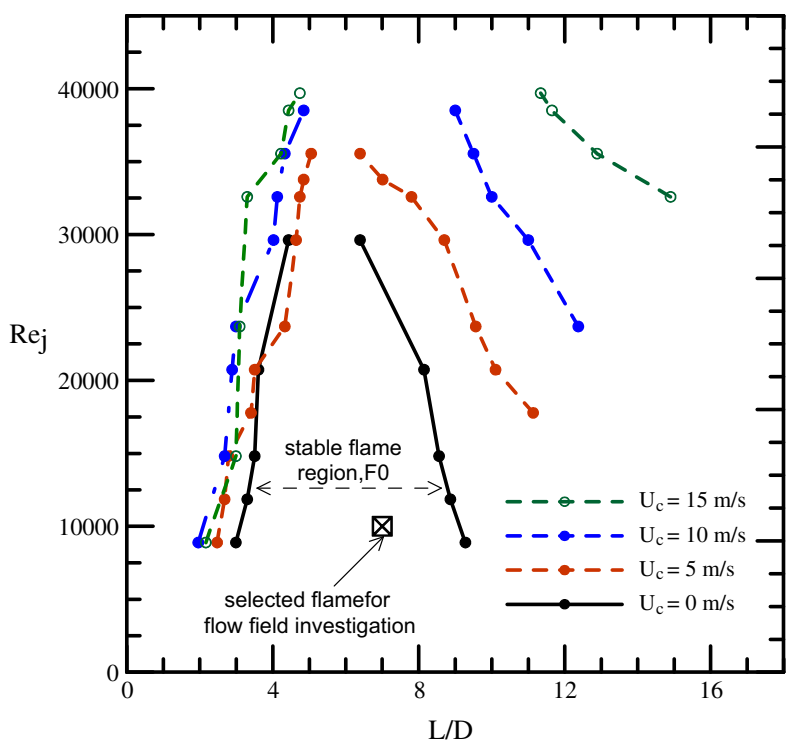

Fig. 2. The stability limits of the $C F C N$ flames with and without co-flow at jet equivalence ratio $\left(\Phi_{j}=2\right)$, and the selected flame for flow field measurments. with and without co-flow, show that the flames are stable between two limits of $L / D$, as shown in Fig. 2. Short distance of $L / D$ refers to flames close to non-premixed flame conditions, while longer distance of $L / D$ refers to a flame close to being fully premixed. The first short distance represents the lower end of mixing between the air and fuel and the second longer distance represents the upper end of mixing. This means that the flame extinguishes on approaching fully premixed at longer value of $L / D$, and approaching non-premixed at shorter $L / D$. Thus partially premixed flames are more stable than premixed or non-premixed flames. This is consistent with previous measurements [16].

The stability data for a jet equivalence ratio of $2\left(\Phi_{j}=2\right)$ without co-flow and several values of co-flow velocity are shown in Fig. 2. The curves on the left hand side of the graph represent the extinction at the lower limit of $L / D$ while those at the right hand side represent the upper limit of $L / D$. The flames are attached to the burner mouth and are stable within the gap between the upper and lower limits of $L / D$, as indicated in Fig. 2 by stable flame region. The results show that the stability is greatly improved by adding co-flow, where the span between these two limits increases with co-flow. This improvement in the stability limits is enhanced by increasing the co-flow velocity. The curves at the lower limits of $L / D$ show some improvement with increasing co-flow velocity while the improvements are much more at the upper limit of $L / D$ approaching premixing limits. Increasing the jet Reynolds number $R e_{j}$, leads to a reduction of the stable flame region. So, a continuous increase of $R e_{j}$ should lead to a critical point above which the flame is extinguished. This critical point depends on the co-flow velocity. It seems to be shifted toward the fully premixed mixture with increasing co-flow velocity. A similar trend has been observed by Meares and Masri [20]. This reduction in the stability region is due to the turbulence effect, which leads to strong turbulencechemistry interaction.

Based on the above stability characteristics of this burner, three flames are selected for the further investigation using the PIV technique; one without co-flow (F0), and the other two flames with co-flow velocities of $5 \mathrm{~m} / \mathrm{s}$ (F5) and $10 \mathrm{~m} / \mathrm{s}$ (F10). The three cases are at the same partial premixing level, where $L / D=7$, with jet Reynolds number $=10,000$, and jet equivalence ratio of 2 . The 
Table 1

The main parameters of the investigated flames.

\begin{tabular}{|c|c|c|c|c|c|c|}
\hline Flames & Co-flow, $V_{c}(\mathrm{~m} / \mathrm{s})$ & Jet velocity, $V_{j}(\mathrm{~m} / \mathrm{s})$ & Jet equivalence ratio $\left(\Phi_{j}\right)$ & Jet Reynolds $\left(R e_{j}\right)$ & Air jet velocity, $V_{a}(\mathrm{~m} / \mathrm{s})$ & Fuel jet, velocity, $V_{f}(\mathrm{~m} / \mathrm{s})$ \\
\hline F0 & 0 & 16.88 & 2 & 10,000 & 82.04 & 4.74 \\
\hline F5 & 5 & & & & & \\
\hline F10 & 10 & & & & & \\
\hline
\end{tabular}

main parameters of these flames are listed in Table 1 , and their positions relative to the stability limits are shown in Fig. 2.

Fig. 3 illustrates the flame appearance for the flames F0, F5, and F10. With the gradual increase of the co-flow velocity, the flame changes from a long flame which resides inside the cone to a broad short structure with the flame sitting on the lip of the conical nozzle. Increasing the co-flow velocity stretches the flame towards the nozzle walls. The flame expansion with co-flow explains the higher stability limit as compared to the flames without co-flow. The gradual increase of the flame expansion due to the increase of the co-flow velocity suggests a local change of the flow field structure at the lip of the cone with likely creation of a recirculation zone that keeps the flame stable and attached to the burner lip. The flow field measurements presented below provides more details on the flame structure and stability.

\subsection{Flow field structure}

The flow field structures were measured for the selected flames at the exit of the conical nozzle. The PIV images are captured at the conical nozzle exit with an image height of $160 \mathrm{~mm}$. A total 500 stereo PIV images were recorded in each flame in order to calculate the mean and rms of the velocity. Figs. 4-6 show the results for the selected flames F0, F5 and F10. The data presented represent the mean velocity vectors overlaid with the streamlines. The third velocity component $(w)$ is not illustrated for clarity as this component did not show a significant value or sensitivity to co-flow velocity.

Typical features of the flow field for the partial premixed flames without co-flow, Flame F0, are shown in Fig. 4. The velocity field shows a typical jet profile with no recirculation zone at the nozzle exit. This is consistent with the visual observations of the flames (Fig. 3). In addition, the burner head remains relatively cold, which indicates that at least there is no recirculation of the hot products inside the nozzle. On the other hand, air entrainment into the conical nozzle may be expected and this may also responsible in keeping the nozzle head relatively cold. As indicated, the velocity vectors do not show the expected outward shift. On the contrary, a large entrainment from the surrounding atmosphere is directed toward the flame boundaries over the entire flame length, as can be seen from outer stream lines pattern along the flame length.

The flame appearance is altered by the existence of the co-flow as shown in Fig. 3 above. With a co-flow, the flame width is

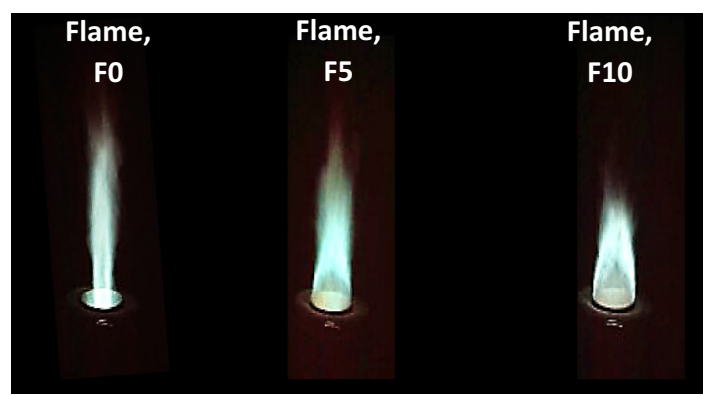

Fig. 3. Photos of partial premixed flames with three co-flow velocities $(0,5,10 \mathrm{~m} / \mathrm{s})$.

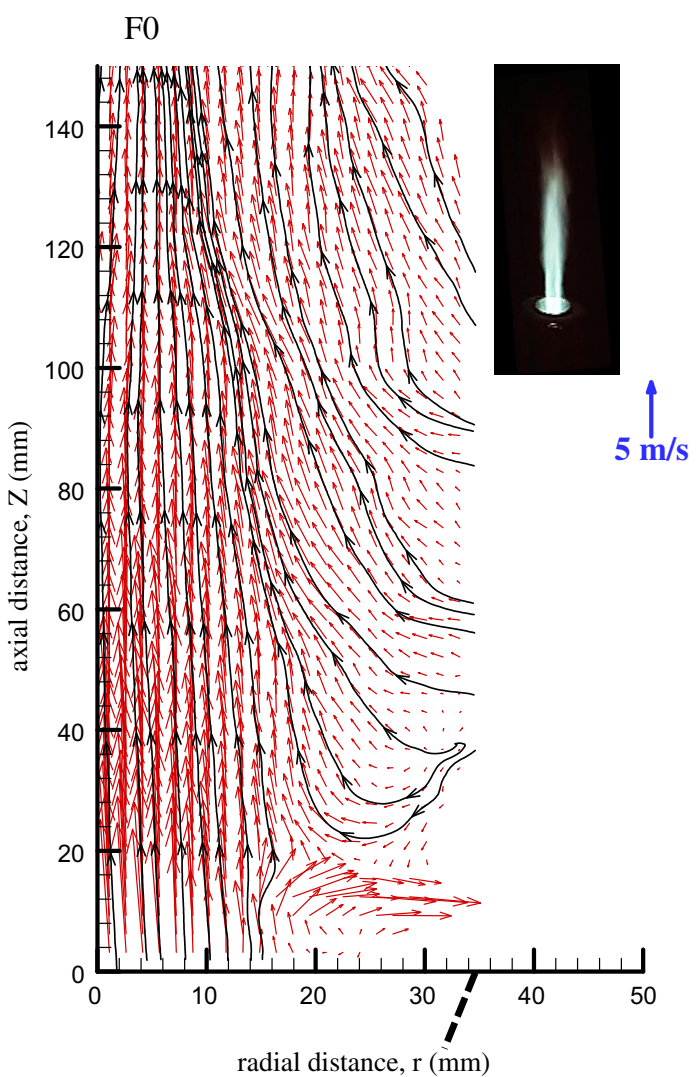

Fig. 4. Velocity vectors overlaid with the streamlines of flame F0 (alternate rows of velocity vectors have been removed for clarity, and the flame photo, dashed line at $x$ axis represents the nozzle wall.

increased and extended to the nozzle wall. In addition, the visible flame length is decreased with increasing the co-flow velocity. These observations for the flame appearance with co-flow is supported and explained by investigation the flow field of flame F5, shown in Fig. 5. The velocity vectors show that a co-flow velocity of $5 \mathrm{~m} / \mathrm{s}$ leads to the formation of two vortices immediately at the nozzle rim, inner and outer vortices, and these vortices surround the central main jet. The average location of the inner vortex is located at $r=18$ and $30 \mathrm{~mm}$, which means this vortex is located within the span of the nozzle radius. The outer vortex, which is larger than the inner, is located at the stream of the co-flow, this vortex is formed due to the entrainment effect of the flame jet. As indicated by the streamlines, that these two vortices enhanced the flux exchange between the co-flow and the flame jet within the early region of the flame. A shear layer exists between the main flame jet and the inner vortex. Through this shear layer, hot combustion products are being continuously mixed with fresh fuel-air mixture, combustion is stabilized and the flame tends to reside there. At the same time, the formation of inner vortex increases the recirculation of the hot products toward the flame root within the nozzle zone which improve the flame stability. At longer axial distances the velocity vectors of the flame jet show a slight outward shift and this matches the flame appearance, which does 
F5

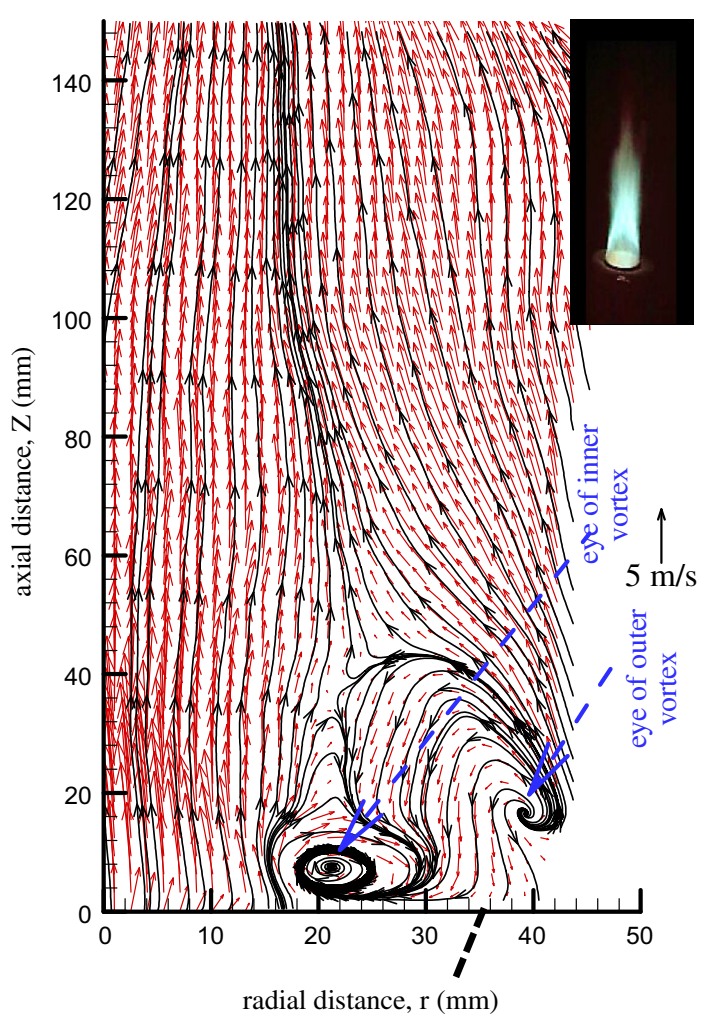

Fig. 5. Velocity vectors overlaid with the streamlines of flame F5 (alternate rows of velocity vectors have been removed for clarity, and the flame photo, dashed line at $x$ axis represents the nozzle wall.

not exist in the flames without a co-flow. However, further downstream this outward shift starts to cease at the flame tip with more co-flow entrainment towards the flame jet.

With further increase of the co-flow velocity to $10 \mathrm{~m} / \mathrm{s}$, the flow field of flame F10 is illustrated in Fig. 6. As indicated, the flow pattern is seen to possess qualitatively the same trend with those previously examined for flame F5 (Fig. 5). However, due to the higher co-flow velocity the strength of the two vortices at the nozzle exit is increased, in terms of their size and circulation. In addition to the increased strength, the location of the inner vortex is slightly shifted toward the flame centerline, which also enhances the flame stability. The formation of such a large vortex in the flow stream acts as a bluff body stabilizer, but with the backflow toward the flame root inside the nozzle. Finally the formation of large vortex means the formation of large zero velocity path line at the early region of the flame, which leads to more improvement of the stability limits. Moreover, the velocity vectors of the main flame jet show a more outward motion in the region of vortex formation. This outward shift steadily decreases with further axial distance and with the more inward shift of the outer co-flow stream, as indicated by the stream lines pattern. Fig. 7 shows the progressive variation of the reversed flow region with the axial distances of both flames F5 and F10. This region is bounded by tracing the location of the zero axial velocity of the inner and outer vortices. As co-flow velocity increases, the width of this zone increases and extends further downstream. This explains the why the flame becomes more stable at higher co-flow velocity.

Fig. 8 illustrates the radial profiles of the mean axial velocity at various axial locations for the three flames. In all the flames, at $Z=10 \mathrm{~mm}$ from the nozzle exit, the velocity profile reveals the presence of a central peak velocity within the flame jet, and the velocity is gradually decreased in the outward direction.
F10

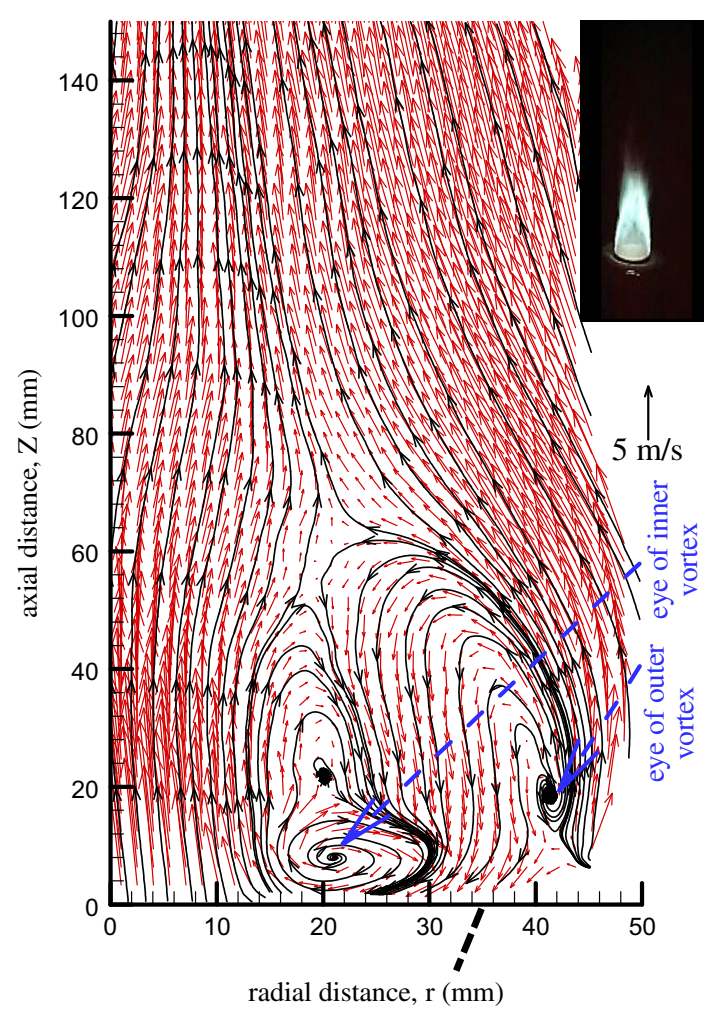

Fig. 6. Velocity vectors overlaid with the streamlines of flame F10 (alternate rows of velocity vectors have been removed for clarity, and the flame photo, dashed line at $x$ axis represents the nozzle wall.

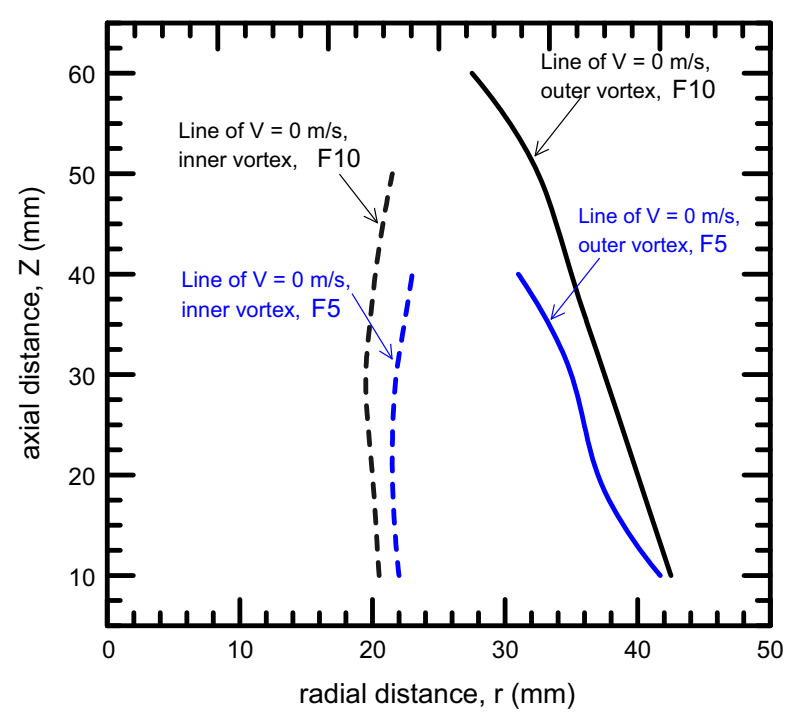

Fig. 7. Reversed flow regions of flames F5 and F10.

Negative velocity regions for the flames with co-flow, Fig. $8 \mathrm{~b}$ and c are observed at the flame boundaries. These negative velocity regions are attributed to the backflow associated with the vortex, at the outer end of this negative velocity region the velocity starts again to increase, and this is due the co-flow stream (see Fig. 7). The negative velocity region has a peak value, and this peak value is higher with higher co-flow velocity, which indicates the 

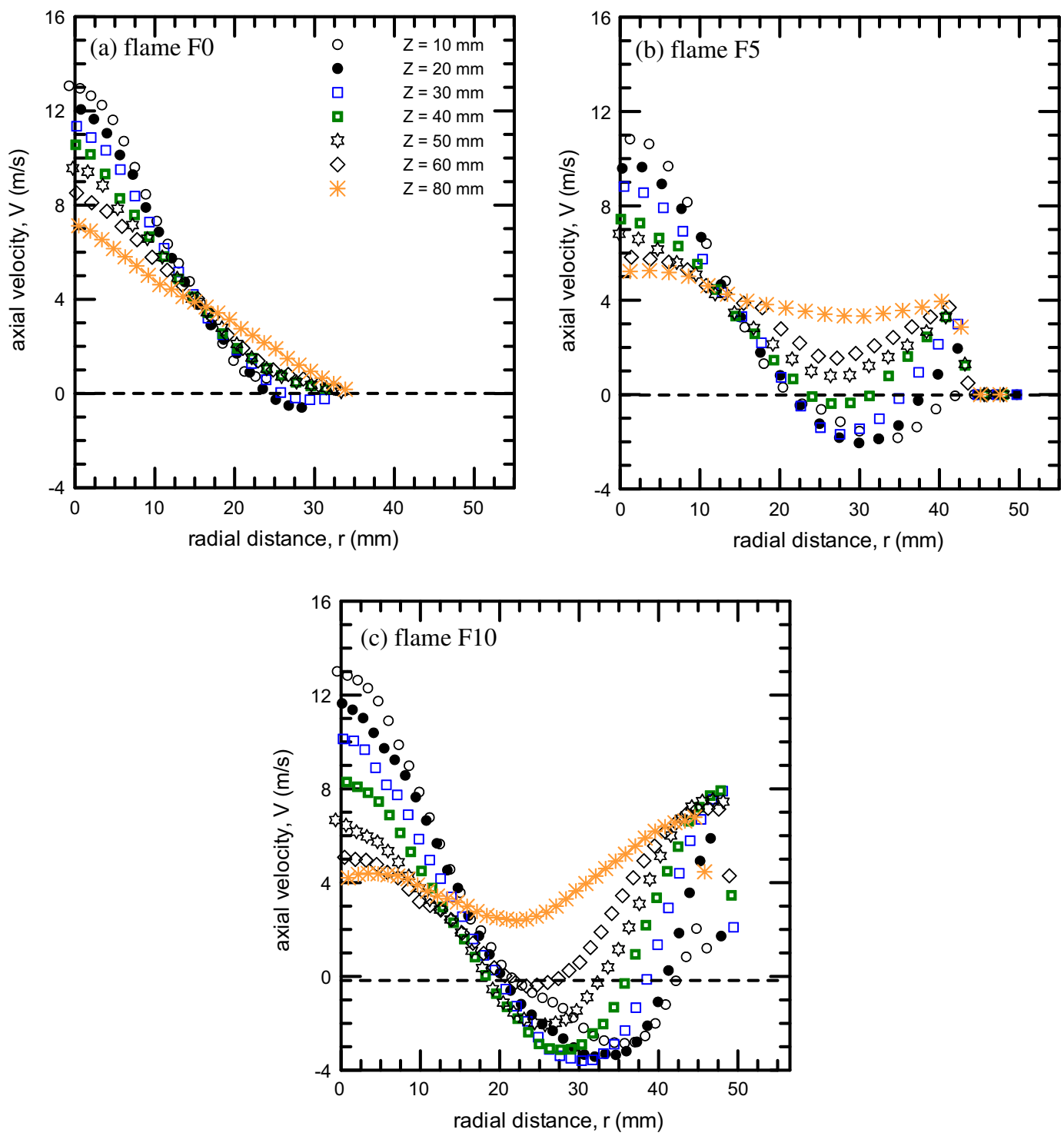

Fig. 8. Radial profiles of mean axial velocity at various axial locations, (a) flame F0, (b) flame F5, (c) flame F10.

increased vortex strength. However, further downstream, the profiles show a steady reduction in the peak velocity of the flame jet but at different rates. With this reduction in the peak velocity, the negative velocity is progressively decreased with a shifting of its peak toward the flame centerline. The size of the reversed flow region gradually declines until the velocity is again all in the positive direction. The reversed flow region is extended for a longer axial distance with the higher co-flow velocity.

The axial velocity profiles (centerline velocity $V_{c e n}$ normalized by the nozzle exit velocity $V_{o}$ ) along the flames centerline are illustrated in Fig. 9. As shown, for flame F0 the centerline declines along the entire flame length, and this is due to the flame expansion. However, for flame F5, $V_{\text {cen }}$ decreases but at a slightly higher rate. At an axial distance of $60 \mathrm{~mm}$, the rate of velocity reduction becomes very low and this recovery in centerline velocity is due to the merging of the co-flow jet with the main flame jet beyond the vortices region and more entrainment at downstream axial distances. Moreover, for flame F10, first it shows a steeper centerline decline (due to large vortex size and more flame stretching) extending to an axial distance of nearly $70 \mathrm{~mm}$, where the centerline velocity starts to increase again due to entrainment and merging of higher velocity co-flow annular jet.

The radial profiles of the axial rms velocities, $V_{r m s}$, at some selected vertical positions are shown in Fig. 10 for the three flames. As shown in Fig. 10a, at early vertical positions $(Z=20 \mathrm{~mm})$ of flame F0, the $V_{\text {rms }}$ profile shows a peak at the flame brush similar to jet flow (nearly at $r=10 \mathrm{~mm}$ ) and this peak position corresponds to that of the gradient in velocity profile (Fig. 8a). This effect is gradually suppressed at further vertical positions of $Z=60 \mathrm{~mm}$, with a higher increase in rms at the flame jet boundaries. The profiles of $V_{r m s}$ for flame F5 show the same peak at radial location $(r=10 \mathrm{~mm})$ is still noticeable, but due to the formation of the vortex at the nozzle exit, a damping of the turbulence level is recorded with a steep increase in $V_{\text {rms }}$ at the co-flow boundaries; see the limits of the reversed flow region at $Z=40 \mathrm{~mm}$. The larger the vortex size, the larger damping effect of the $V_{r m s}$. This could be explained by close inspection of the $V_{r m s}$ radial profile for flame F10 (Fig. 10c) at $Z=40 \mathrm{~mm}$. In addition, the peak $V_{r m s}$ value at $r=10 \mathrm{~mm}$ recorded for both flames F0 and F5 is changed into a Gaussian profile in flame F10 $V_{r m s}$ profile at $Z=20$ and $40 \mathrm{~mm}$. 


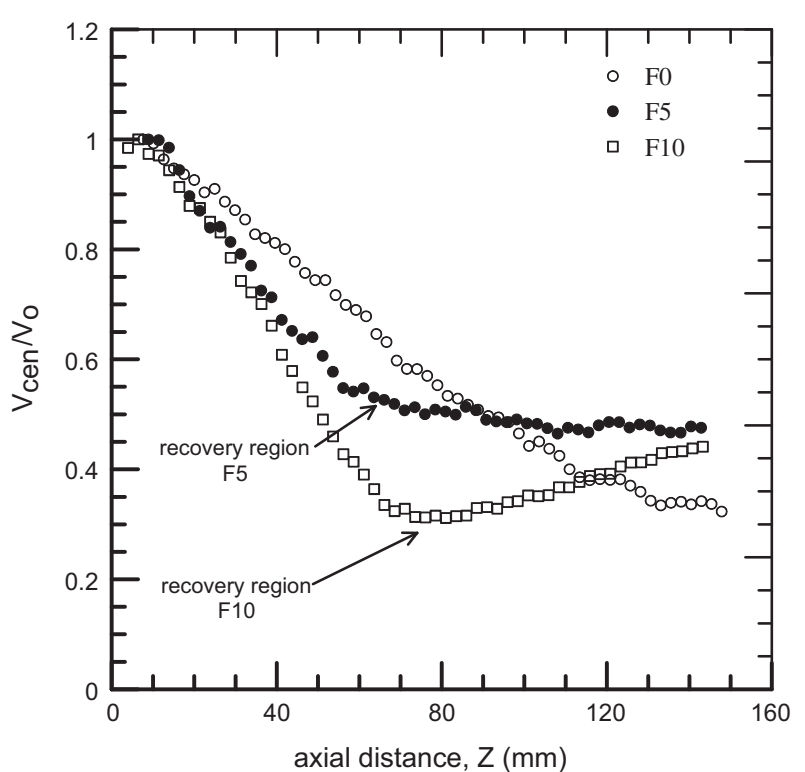

Fig. 9. Axial center line velocity normalized by the exit velocity for flames F0, F5, and F10.

The reduction of the velocity fluctuation levels at the flame brush and or at the flame boundaries should provide the co-flow flames with better stability conditions.

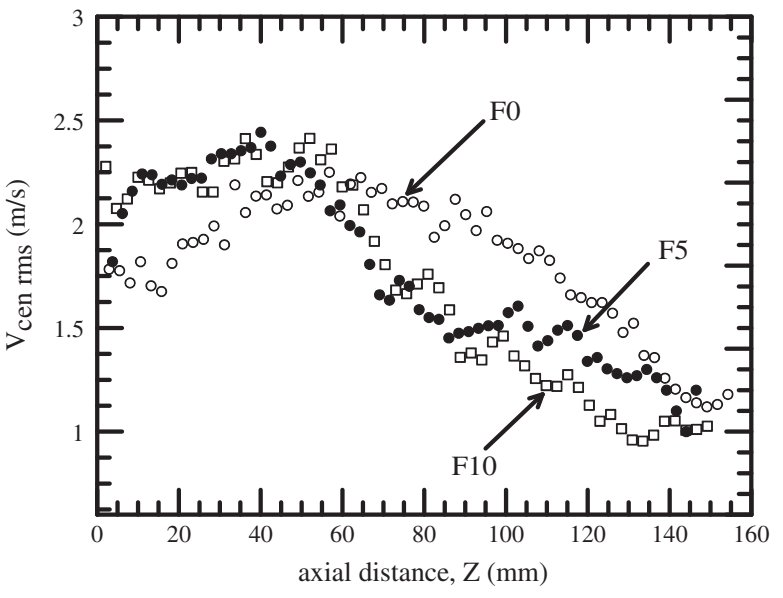

Fig. 11. Axial centerline velocity fluctuations, $V_{\text {cen } r m s}$ of flames F0, F5, and F10.

Moreover, the centerline velocity rms distributions for the investigated three flames are plotted in Fig. 11. As already mentioned, at early axial distance from the nozzle exit, the center line velocities decline, but with different rates (Fig. 9). This is associated with an increase in velocity fluctuations, but with a higher rate of increase with the co-flow flames, see Fig. 11. For all the flames, rms profiles increase, reaching a peak centerline velocity fluctuation at further downstream axial distances. However, the
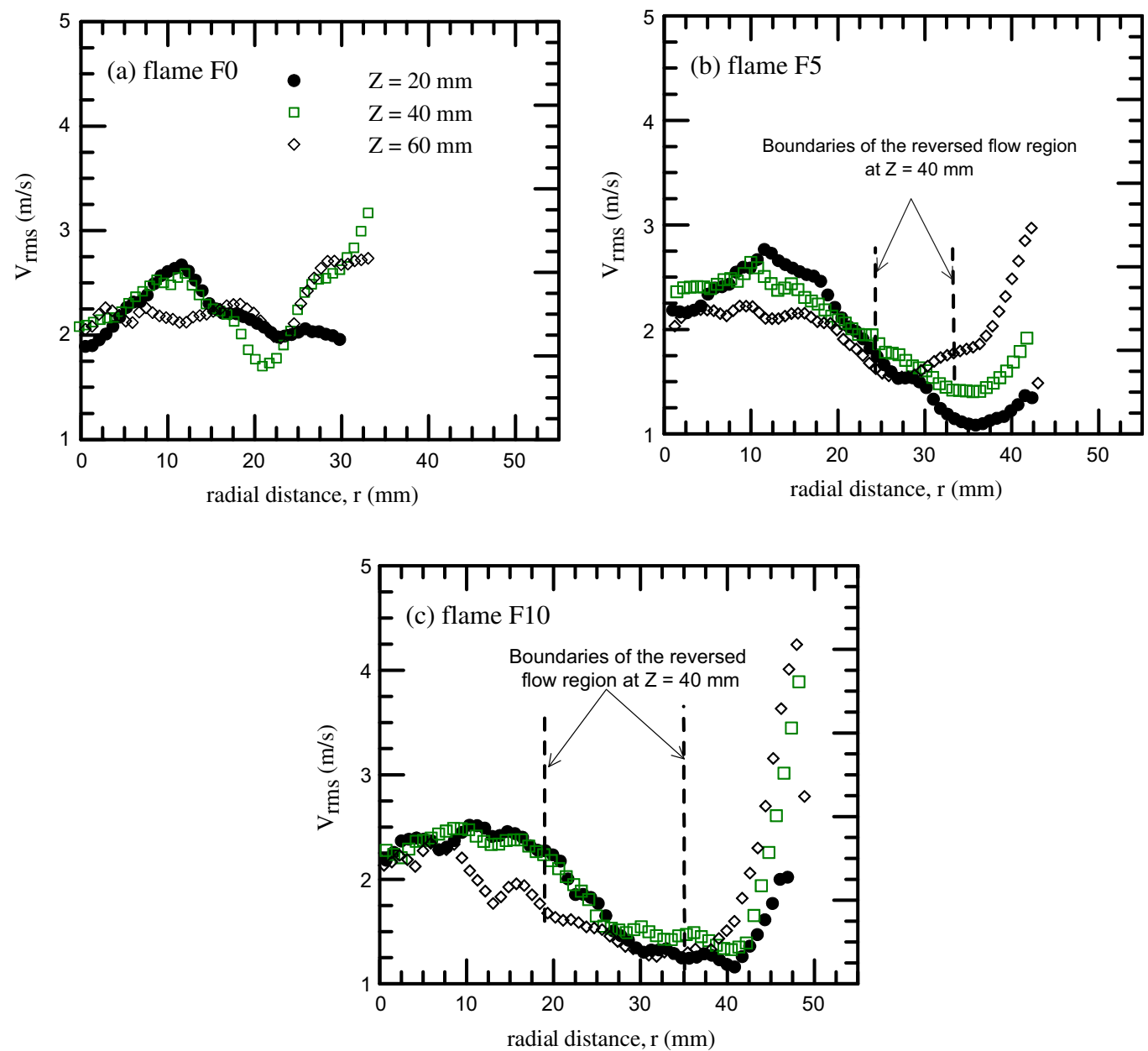

Fig. 10. Radial distribution of the axial velocity fluctuations, $V_{r m s}$, at different axial positions at $Z=20,40$, and 60 mm, for (a) flame F0, (b) flame F5, and (c) flame F10. 
flame without co-flow exhibits a higher rms at the centerline than those flames with co-flow after the peak rms axial location (around $Z=45 \mathrm{~mm}$ ), with much less rms at the flame tip for flame F10. This may be due to the sustained centerline velocity at the flame tip with co-flow.

\section{Conclusions}

In this work, the effect of the co-flow velocity on the stability limits of partial premixed methane flame issuing from a concentric flow conical nozzle burner have been investigated and discussed. The degree of partial premixing is well controlled with a simple technique to cover a wide range between fully premixed and non-premixed conditions. The flame stability map of the flames with and without co-flow velocity is presented and the two extinction limits for the high and low degree of partially premixed are presented. The turbulent flow field of three flames at $0,5,10 \mathrm{~m} / \mathrm{s}$ co-flow velocity are investigated using stereo PIV in order to explore the improvement of the flame stability due to the existence of the co-flow.

The measurements show that the flame is highly stabilized by the co-flow, due to the generation of two vortices at the nozzle exit. The flames with co-flow expand due to these vortices and are stabilized at the lip of the cone while the flame length is decreased. The level of partial premixing significantly affects the stability of the flames both with and without co-flow. This leads to the conclusions that partially premixed flames are more stable than premixed and non-premixed cases with an optimum degree of partial premixing. The increase of the co-flow velocity leads to further improvement of the stability. This effect is more obvious when the flames approaches fully premixed as compared to those near non-premixed conditions. In addition, the velocity fluctuations are suppressed at the shear layer with the co-flow.

The effect of co-flow on the flame structure and stability is essential for the development of industrial burners. Other effects of the varying the equivalence ratio and fuel type are also required and should be investigated. Further investigations of the events occurring inside the nozzle using high speed simultaneous OH-PLIF/PIV measurements using a transparent quartz nozzle are recommended.

\section{Acknowledgments}

The present work was conducted at clean combustion research center, CCRC, King Abdullah University for Science and Technology,
KAUST, in cooperation with Cairo and Helwan Universities, Cairo, Egypt.

\section{References}

[1] S. Aggarwal, Extinction of laminar partially premixed flames, Prog. Energy Combust. Sci. 35 (2009) 528-570.

[2] M. Mansour, R. Bilger, R. Dibble, Raman/Rayleigh and Mie scattering measurements in a reverse flow reactor close to extinction, Proc. Combust. Inst. 22 (1989) 711-719.

[3] M. Mansour, Studies of Turbulent Hydrocarbon Flames in a Reverse Flow Reactor, PhD Diss., Sydney University, Sydney, Australia, 1990.

[4] M. Mansour, A concentric flow conical nozzle burner for highly stabilized partially premixed flames, Combust. Sci. Technol. 152 (2000) 115-145.

[5] R. Barlow, J. Frank, Effects of turbulence on species mass fractions in methane/ air jet flames, Proc. Combust. Inst. 27 (1998) 1087-1096.

[6] A. Masri, Y. Al-Abdeli, Stability characteristics and flow fields of turbulent nonpremixed swirling flames, Combust. Theory Model. 7 (4) (2003) 731-766.

[7] R. Schefer, D. Wicksall, A. Agrawal, Combustion of hydrogen-enriched methane in a lean premixed swirl-stabilized burner, Proc. Combust. Inst. 29 (2002) 843851.

[8] J. Ji, J. Gore, Flow structure in lean premixed swirling combustion, Proc. Combust. Inst. 29 (2002) 861-867.

[9] P. Kalt, Y. Al-Abdeli, A. Masri, R. Barlow, Swirling turbulent non-premixed flames of methane: flow field and compositional structure, Proc. Combust. Inst. 29 (2002) 1913-1919.

[10] S. Starner, R. Bilger, Characteristics of a piloted diffusion flame designed for study of combustion turbulence interactions, Combust. Flame 61 (1985) 2938.

[11] A. Masri, R. Dibble, R. Barlow, The structure of turbulent non-premixed flames revealed by Raman-Rayleigh-lif measurements, prog, Energy Combust. Sci. 22 (1996) 307-362.

[12] Y. Chen, N. Peters, G. Schneemann, N. Wruck, U. Renz, M. Mansour, The detailed flame structure of highly stretched turbulent premixed methane-air flames, Combust. Flame 107 (1996) 223-244.

[13] I. Esquiva-Dano, H. Nguyen, D. Escudie, Influence of a bluff body's shape on the stabilization regime of non-premixed flames, Combust. Flame 127 (2001) 2167-2180.

[14] A. Masri, B. Dally, R. Barlow, C. Carter, The structure of the recirculation zone of a bluff-body combustor, Proc. Combust. Inst. 25 (1994) 1301-1308.

[15] B. Yan, B. Li, E. Baudoin, C. Liu, Z. Sun, Z. Li, X. Bai, M. Aldén, G. Chen, M. Mansour, Structure and stabilization of low calorific value gas turbulent partially premixed flames in a conical burner, Exp. Therm. Fluid Sci. 34 (2010) 412-419.

[16] F. El-Mahallawy, A. Abdelhafez, M. Mansour, Mixing and nozzle geometry effects on the flame structure and stability, Combust. Sci. Technol. 1179 (2007) 249-263.

[17] M. Mansour, A. Elbaz, M. Samy, The stabilization mechanism of highly stabilized partially premixed flames in concentric flow conical nozzle burner, Exp. Therm. Fluid Sci. 43 (2012) 55-62.

[18] E. Baudoin, X. Bai, B. Yan, C. Liu, R. Yu, A. Lantz, S. Hosseini, B. Li, A. Elbaz, M. Sami, Z. Li, R. Collin, G. Chen, L. Fuchs, M. Aldén, M. Mansour, Effect of partial premixing on stabilization and local extinction of turbulent methane/air flames, Flow Turbul. Combust. 90 (2013) 269-284.

[19] A. Elbaz, Early structure of LPG partially premixed conically stabilized flames, Exp. Therm. Fluid Sci. 44 (2013) 583-591.

[20] S. Meares, A. Masri, A modified piloted burner for stabilizing turbulent flames of inhomogeneous mixtures, Combust. Flame 161 (2014) 484-495. 